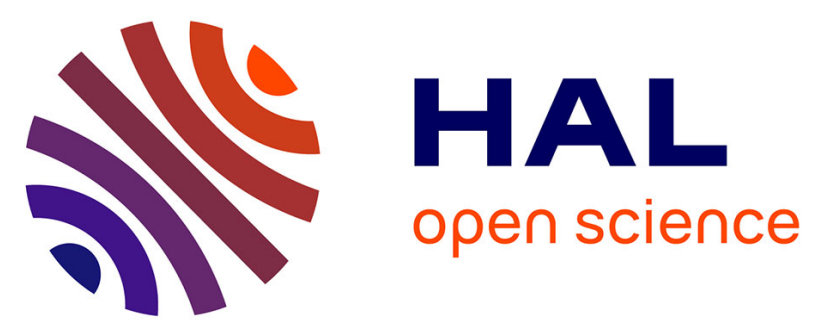

\title{
Do Curve Characteristics Influence Stenosis Location and Occurrence of Radicular Pain in Adult Degenerative Scoliosis?
}

E. Ferrero, M. Khalifé, L. Marie-Hardy, N. Regnard, A. Feydy, C. Garreau de Loubresse, S. Zakine, P. Guigui

\section{To cite this version:}

E. Ferrero, M. Khalifé, L. Marie-Hardy, N. Regnard, A. Feydy, et al.. Do Curve Characteristics Influence Stenosis Location and Occurrence of Radicular Pain in Adult Degenerative Scoliosis?. Spine Deformity, 2019, 7, pp.472 - 480. 10.1016/j.jspd.2018.09.010 . hal-03484395

\section{HAL Id: hal-03484395 https://hal.science/hal-03484395}

Submitted on 20 Dec 2021

HAL is a multi-disciplinary open access archive for the deposit and dissemination of scientific research documents, whether they are published or not. The documents may come from teaching and research institutions in France or abroad, or from public or private research centers.
L'archive ouverte pluridisciplinaire HAL, est destinée au dépôt et à la diffusion de documents scientifiques de niveau recherche, publiés ou non, émanant des établissements d'enseignement et de recherche français ou étrangers, des laboratoires publics ou privés.

\section{(c) (1) $\$$}

Distributed under a Creative Commons Attribution - NonCommerciall 4.0 International 


\section{Manuscript Title}

Do Curve Characteristics influence Stenosis Location and Occurrence of Radicular Pain in Adult Degenerative Scoliosis?

\section{Authors}

E. Ferrero, $\mathrm{MD}^{1}$, MSc; M. Khalifé, $\mathrm{MD}^{1}$; L. Marie-Hardy, $\mathrm{MD}^{1}$; N. Regnard, MD²; A. Feydy, MD²; C. Garreau De Loubresse, $\mathrm{MD}^{1}$; S. Zakine, $\mathrm{MD}^{3} ; \mathrm{P}$. Guigui, $\mathrm{MD}^{1}$

1: Orthopedic Surgery department, Hôpital Européen Georges-Pompidou, APHP, Paris V University, 20 rue Leblanc, 75908 Paris Cedex 15, FRANCE

2: Radiology department, Hôpital Cochin, APHP, Paris V University, 75014 Paris, FRANCE

3: Orthopedic Surgery department, Clinique les Maussins, 75019 Paris, FRANCE

\section{Correspondence}

Ferrero, Emmanuelle

Email: Emmanuelle.ferrero@gmail.com

Phone: +331560932 72; Fax: +33156092395

Hôpital européen Georges-Pompidou, APHP, Paris V University, 20 rue Leblanc, 75908 PARIS

Cedex 15

This manuscript does not contain any copyrighted material.

The authors report no conflict of interest concerning the materials or methods used in this study or the findings specified in this paper.

Funding: no grant support received.

IRB Approval was granted for this study at all participating sites. 
Do Curve Characteristics influence Stenosis Location and Occurrence of Radicular Pain in Adult Degenerative Scoliosis?

\begin{abstract}
Study design. Retrospective cohort

Objective. The aim of this study was to describe the various locations of spinal stenosis (LSS) in lumbar scoliosis (DLS) and its related clinical symptoms.
\end{abstract}

Introduction. Adult with lumbar scoliosis often present with pain and disability. Association of scoliosis and stenosis is not rare, but remains sparsely explored. Consequences of scoliosis on stenosis location and treatment remain debated.

Methods. Patients operated for symptomatic LSS with DLS (Cobb angle>20 ${ }^{\circ}$ ) from 2015 to 2016 were included. All patients completed preoperative clinical and neurologic exam. Coronal and sagittal radiographic parameters, rotatory subluxation (RS) and spondylolisthesis were analyzed on fullspine x-rays. CT-scan multiplanar reconstructions were performed to measure central, foraminal and lateral recess stenosis stenosis, from T10 to Sacrum.

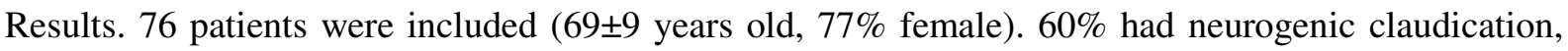
L5 was the most common radicular pain (41\%). Mean Cobb angle was $33 \pm 16^{\circ} .35$ (46\%) patients had coronal malalignment; in $69 \%$, side of the coronal tilt corresponded to side of the concavity of the lumbosacral curve. 60 patients had RS (most frequent level L3L4). In 50\% of the cohort, RS was located at junction between lumbar and lumbosacral curves. In $70 \%(\mathrm{n}=53)$ of the patients, central stenosis occurred at junction between lumbar and lumbosacral curve. Foraminal and lateral stenosis were most frequently observed in the concavity of the distal lumbo-sacral curve. L5 radicular pain was significantly more frequent in case of lumbo-sacral contra-curve and right coronal malalignment. 
Conclusion. LSS is frequent in DLS. Relationships exist between curve characteristics and symptomatic LSS in DLS; especially, concavity of the lumbosacral contra-curve and the junctional level between lumbar curve and lumbosacral contra-curve and. Therefore, accurate analysis of stenosis in ASD seems mandatory, to at least performed decompression; since, perfect planned treatment for stenosis and scoliosis correction might not be always performed, due to patient general health status.

\section{Level 4}




\section{Introduction}

Attention for adult spinal deformity (ASD) has increased over the past decades and great technical advances have been made in scoliosis surgery. However, the evaluation of patients' functional and clinical outcomes became more and more important. Indeed, it has been well demonstrated that sagittal malalignment is significantly correlated with poor outcomes on health related quality of life (HRQL) (1-5).

The association between scoliosis and stenosis has been rarely reported in the literature $(1,6)$. However, this association is frequent, since up to $20 \%$ of the patients with a lumbar spinal stenosis had a scoliosis and $90 \%$ of the patients with lumbar degenerative scoliosis had spinal stenosis $(7,8)$. Nevertheless, even though the clinical presentation differs between patients, neurologic deficits are pretty rare and the symptoms showed a high variability. Smith, in a cohort of 207 scoliosis patients, observed $8 \%$ of lower limbs weakness and $9 \%$ of neurogenic claudication (9). The lack of reports might be explained by the complexity of the anatomical analysis of stenosis associated with lumbar scoliosis. Moreover, this description is not easy to perform since lumbar scoliosis showed a large variability in its origins. Indeed, lumbar ASD may be an evolution of an adolescent idiopathic scoliosis, or may occur de novo with asymmetric disc degeneration and facets joints, with or without global malalignment, rotatory subluxation and spondylolisthesis (10) (Fig. 1). Several hypotheses have been evoked concerning the location of the stenosis in adult scoliosis but remained debated $(1,6-8)$. Nevertheless, in these series intracanalar imaging was not always performed and cohorts were small. While the origin of adult lumbar scoliosis is not fully understood yet, the role of scoliosis in specific anatomical characteristic of lumbar spinal stenosis and its consequences on treatment remains also to be investigated.

The aim of this study was to describe the various locations of spinal stenosis in lumbar scoliosis and attempt to understand the role of the deformity in lumbar spinal stenosis and its relation with the clinical symptoms. 


\section{Methods}

Study

Following institutional review board approval, a prospective monocentric series of consecutive patients, with scoliosis and symptomatic lumbar spinal stenosis (LSS), were analyzed between March 2015 and August 2016.

Patients were included if they had a lumbar scoliosis with an apex located between the L1-L2 disc and L4 or a thoraco-lumbar scoliosis with an apex located between T12 and L1 (old idiopathic or de novo degenerative) with a Cobb angle greater than $20^{\circ}$, a symptomatic lumbar spinal stenosis and complete imaging including lumbar CT scan and fullspine coronal and sagittal x-rays.

Patients with prior spinal surgery, other causes of scoliosis, neurological or vascular disease were excluded.

\section{Data collection}

Usual demographic data (age, gender, body mass index) were reported. Otherwise, leg pain, radicular pain, motor or sensory deficit, neurogenic claudication and walking area were also collected.

All the patients underwent fullspine coronal and sagittal x-rays using a standardized protocol: patients were instructed to adopt a free standing position with horizontal gaze, and fists on clavicle to avoid superimposition of the arms with the spine (11). A single experienced operator performed all the 2D radiographic parameters analysis using Keops software (SMAIO, Lyon, France)(12). In the coronal plane, Cobb angles and transitional vertebrae between the main lumbar curve, the proximal thoracic curve and the lumbosacral contra-curve were analyzed. Global coronal alignment was assessed by C7 coronal tilt (C7CT) defined by the angle between the line joining the center of $\mathrm{C} 7$ and the middle of the sacral endplate, and the vertical reference line in the coronal plane (Fig. 2). Coronal malalignment was defined by a C7CT $>3^{\circ}$ on either side. The level of rotatory subluxation (RS) as well as the type of RS were reported: open or close (Fig. 3) $(13,14)$. Sagittal parameters included, pelvic parameters (pelvic incidence, pelvic tilt and sacral slope), maximal lumbar lordosis (LLmax), maximal thoracic kyphosis (TKmax), and C7 sagittal tilt (C7ST) for the global alignment)(13,15). Sagittal malalignment 
was defined by a C7ST $>7.7^{\circ}$ anteriorly or posteriorly (Fig. 2) (13). Existence of degenerative spondylolisthesis was also recorded.

Stenosis was diagnosed with the use of lumbar CT scanner with multiplanar reconstructions. One experienced spine surgeon and one experienced radiologist performed lumbar canal measurements without any knowledge of the patient clinical symptoms. Central stenosis was assessed on axial slices by using the surface area of the dural sac. Severe central stenosis was defined by a surface area < $70 \mathrm{~mm}^{2}$, a moderate stenosis by a surface between 70 and $100 \mathrm{~mm}^{2}$ (16-18). Foraminal stenosis was defined on sagittal slices by a cranio-caudal or antero-posterior diameter of the foramen $<4 \mathrm{~mm}(19$ 22). Foraminal width was defined by the antero-posterior maximum dimension between the inferoposterior wall of the vertebra and the top of the superior facet of the lower vertebra. Foraminal height was defined by the maximum distance between the inferior margin of the pedicle and the superior margin of the pedicle of the lower vertebra or of the annulus in case of disc bulging (Fig. 4). Lateral stenosis was defined by an antero-posterior distance of the lateral recess $<3 \mathrm{~mm}$, which corresponded to the distance between the most anterior point of the superior articular facet and the posterior margin of the vertebral body $(17,18,21,23)$.

\section{Statistical analysis}

Data were statistically analyzed using Stata software 14.0 (Statacorp, College Station, Texas). A Shapiro-Wilk test was performed to assess data distribution. First, a descriptive analysis was conducted for the deformity and the location of the stenosis (central, lateral and foraminal). Second, the influence of the deformity on the stenosis was analyzed including the role of the concavity, the junctional area between the main curve and the contra-curves, the rotatory subluxation, the presence of degenerative spondylolisthesis and the global malalignment. A p $<0.05$ was considered significant. 


\section{Results}

\section{Clinical presentation}

A total of 76 patients ( $77 \%$ of females) were included, with a mean age of 69 years \pm 9 (49 to 85) and a mean BMI of $26 \mathrm{~kg} / \mathrm{m}^{2} \pm 5$. Sixty percent of the patients suffered from neurogenic claudication. The walking area was $<200 \mathrm{~m}, 200$ to $1000 \mathrm{~m}$ and $>1000 \mathrm{~m}$ in respectively 24,25 and 27 patients. Most of the patients had L5 radiculalgia (41\%). Remaining patients had L4, S1 and L3 radiculalgia in respectively $21 \%, 13 \%$, and $7 \%$ of the cases. However, only $25 \%$ of the patients had a significant motor deficit (motor testing $<3 / 5$ ).

\section{Analysis of the deformity}

In terms of curve types, $72 \%$ had lumbar curves and $28 \%$ had thoraco-lumbar. A right concavity was found in $56 \%$ of the patients. Mean Cobb angle of the main curve was $33 \pm 16^{\circ}\left(20^{\circ}\right.$ to $85^{\circ}$ ) including 42 patients with a Cobb angle greater than $30^{\circ}$. Mean Cobb angle of the proximal thoracic curve was $20 \pm 13^{\circ}$ and $16 \pm 10^{\circ}$ for distal lumbosacral curve. Mean C7CT was $3.3 \pm 3^{\circ}$, with $46 \%(n=35)$ of the patients with a global coronal malalignment. Among these 35 patients, the side of the coronal tilt corresponded to the side of the concavity of the lumbosacral curve in $69 \%$ ( $\mathrm{n}=24,15$ cases on the left side and 9 cases on the right side).

Sixty patients (87\%) had a rotatory subluxation on 1 to 3 levels (Fig. 5). Most common levels for the rotatory subluxation were L3L4 (37\%), L2L3 (23\%) and L4L5 (21\%). In 19\% ( $\mathrm{n}=16)$ of the cases, the rotatory subluxation was at the apex of the main curve and corresponded to an open subluxation. In $18 \%(\mathrm{n}=14)$, a rotatory subluxation was observed at the upper transitional vertebra between the thoracic and the main lumbar curve, including 10 patients with a closed subluxation. In $50 \%(\mathrm{n}=38)$ of the series, a rotatory subluxation was located at the lower transitional vertebra, between the lumbar and the lumbosacral curves, including 36 patients with a closed subluxation.

Sagittal spinopelvic parameters are summarized in Table 1. Mean C7 sagittal Tilt was $6.7 \pm 6.2^{\circ}$, with $39 \%$ of the patients with anterior malalignment. Twenty-two (29\%) patients had degenerative spondylolisthesis, located at L4L5 level in $90 \%$ of the cases and asymmetric in 13 patients on the CT scan. 


\section{Stenosis analysis}

Central lumbar spinal stenosis was found on average over 2 levels. Severe stenosis $\left(<70 \mathrm{~mm}^{2}\right)$ was observed in $72 \%(n=55)$ of the patients and involved one level in $37 \%$ of these cases, 2 levels in $21 \%$ and 3 to 6 levels in the remaining 14\% (Fig. 6). Central stenosis occurred at the junction between main lumbar and distal lumbosacral curve in $70 \%(\mathrm{n}=53)$ of the patients and closed rotatory subluxation occurred at the same level in 33 patients. Ten patients had central stenosis at the junction between the main lumbar and the proximal thoracic curve, including 6 with closed rotatory subluxation at the same level. Central LSS was diagnosed in the concavity of the main lumbar curve in $34 \%(n=26)$ of the patients. Mean values of the canal surface area are reported in Table 2. Patients with degenerative spondylolisthesis had more severe central stenosis $(85 \%$ vs $50 \%, \mathrm{p}=0.01)$ with a mean canal surface area in L4L5 significantly smaller than patients without degenerative spondylolisthesis $\left(52 \pm 20 \mathrm{~mm}^{2}\right.$ vs $85 \pm 47 \mathrm{~mm}^{2}, \mathrm{p}=0.004$ ) (Fig. 7).

Foraminal stenosis was found in $58 \%(\mathrm{n}=44)$ of the patient and was located at the junction between main lumbar and distal lumbosacral curve in 14 cases (10 cases were associated with closed rotatory subluxation) (Table 3). Foraminal stenosis was most frequently observed in the concavity of the lumbosacral curve $(29 \%, \mathrm{n}=22)$, followed by the concavity of the main lumbar curve $(22 \%, \mathrm{n}=17)$. Lateral stenosis concerned $58 \%(n=44)$ of the patients. It was most frequently observed in the concavity of the lumbosacral curve $(36 \%, n=27)$ (Table 4). However, Cobb angle of the main curve and global coronal tilt were not correlated to any of the stenosis (respectively, $R=0.211, p>0.55$ and $\mathrm{R}=0.306, \mathrm{p}>0.54)$.

\section{Relationship between stenosis and symptoms}

Among the 55 patients with severe central stenosis, 34 had neurogenic claudication. The side of the radiculalgia frequently corresponded to the side of the coronal tilt i.e. $63 \%$ of the patients with a right radiculalgia had a right coronal shift and $72 \%$ of the patients with a left radiculalgia had left coronal shift $(\mathrm{p}=0.02)$. In a more detailed analysis, we observed that $100 \%$ of the 9 patients with a right coronal tilt and right concavity of the lumbosacral curve had right L5 or S1 radiculalgia and that $60 \%$ 
of the 15 patients with left coronal tilt and left concavity of the lumbosacral curve had left L5 or S1 radiculalgia.

All the 20 patients with L4L5 degenerative spondylolisthesis had a central stenosis. Among these 20 patients, 16 had severe stenosis and all had associated radiculalgia.

L3 radicular pain was significantly associated with severe central stenosis in L2L3 $\left(105 \pm 38 \mathrm{~mm}^{2} \mathrm{vs}\right.$ $\left.60 \pm 23 \mathrm{~mm}^{2}, \mathrm{p}=0.04\right)$. L4 left radicular pain was associated to L3L4 severe central stenosis $(83 \pm 34$ $\mathrm{mm}^{2}$ vs $62 \pm 29 \mathrm{~mm}^{2}, \mathrm{p}=0.04$ ) and to L4L5 closed rotatory subluxation in $58 \%$ of the cases. L4 right radiculalgia was associated with L4L5 right foraminal stenosis (in the concavity) and to L3L4 open RS in $64 \%$ of the cases (Fig. 8). L5 radicular pain was associated with L4L5 central stenosis and with foraminal L5S1 and lateral L5 stenosis in lumbosacral curve concavity. Interestingly, less than $50 \%$ of the lateral stenosis were symptomatic. 


\section{Discussion}

Few studies have analyzed relationships between lumbar spinal stenosis and overall spinal alignment in patients with lumbar scoliosis. The present series of 76 patients highlighted that radio-anatomical knowledge of stenosis in adult scoliosis is of upmost importance, since there exist relationships between curve characteristics and spinal canal stenosis. Even though surgical ASD treatment has improved, in some cases, pain relief failed after surgery; it might be related to the lack of awareness of the stenosis. Burton et al. demonstrated that persistent pain, present in $60 \%$ of their patients, was due to the lack of treatment of foraminal stenosis (24). This emphasized the importance of an accurate anatomical analysis of the compression area in scoliotic patients as performed in the current study.

In the present series, most patients had a moderate curve (mean Cobb of $33^{\circ}$ ) and a coronal malalignment was found in $46 \%$ of the patients, which was higher than other series (25\% to $35 \%$ ) $(3,7)$. Nevertheless, all authors agreed that an important global coronal malalignment is clearly associated with poor patient's outcomes. An important finding was that in $69 \%$ of the cases, coronal malalignment was associated to the side of the lumbosacral contra-curve (i.e. C7CT shifted to the convex side of the main lumbar curve) (Fig. 9). Bao et al. in a series of 284 ASD patients, has recently demonstrated that these patients were more at risk of postoperative coronal imbalance, which is associated with poor outcomes (25). Therefore, considering the lumbosacral contra-curve is essential for the correction of the deformity. Moreover, more than half of the present cohort had rotatory subluxations (79\%), most frequently at L3L4 levels which corresponded to the junction between the lumbar and the lumbosacral contra-curve. Therefore, it is also important to diagnose and treat rotatory subluxations, which is often associated with stenosis and since it is a marker of curve evolution and poor outcomes (26-28).

Results of this study also highlighted that severe central canal stenosis was frequent in symptomatic ASD patients since it was present in $72 \%$ of the cohort and involved 2 levels or more in $2 / 3$ of the cases. Interestingly, the junction between lumbar and lumbosacral contra-curve was the site of the central stenosis in $70 \%$ of the cases and was frequently associated with a rotatory subluxation at the same level (in 33 patients). As reported by other authors, RS at the junction between lumbar and 
lumbosacral curves were frequently associated with stenosis and were sometimes the only level of compression (7).

Concavity of the lumbosacral contra-curve was also a major location of neural compression, since it was associated with foraminal stenosis in $59 \%$ of the cases. Lateral stenosis was also commonly observed in the concavity of lumbosacral contra-curve. Likewise, Morin and Deburge reported high rates of stenosis in the concavity of the lumbosacral contra-curve, site of severe osteoarthritis (7). Another factor frequently responsible for central canal stenosis was degenerative L4L5 spondylolisthesis. L4L5 spondylolisthesis corresponded to L4L5 stenosis in all cases. It corresponded to severe L4L5 stenosis in $80 \%$ of the cases, which was always associated with L4 or L5 radiculalgia. Similarly, Morin and Deburge described a degenerative spondylolisthesis in $25 \%$ of their scoliotic patients and suggested that the associated stenosis was an epiphenomenon (7). Lateral listhesis in these ASD patients probably increased the severity of central and foraminal stenosis.

The concordance between radiologic findings and symptoms found in our study is also of particular importance. Analysis of nerve roots compression and radiculalgia revealed that L5 was the most common painful root $(41 \%)$. The side of radiculalgia was also significantly associated with the side of the coronal tilt i.e. with the concavity of the lumbosacral contra-curve, highlighting the relationship between radiculalgia and lateral or foraminal stenosis of the contra-curve. Regarding the territory of the radiculalgia, L3 radiculalgia was significantly associated to L2L3 central stenosis which could be explained by the frequency of closed RS at this level. In the same way, L4 left radiculalgia was related to L3L4 central stenosis or L4L5 closed rotatory subluxation, corresponding to a narrowing of L4 left foramen. In contrast, L4 right radiculalgia was mostly associated to L3L4 open rotatory subluxation, frequently associated with a stretching of L4 roots and L4L5 foraminal stenosis in the concavity of the lumbosacral curve. Finally, L5 radicular pain was associated with L4L5 central stenosis mostly due to L4L5 degenerative spondylolisthesis and L5 foraminal or L5 lateral stenosis in the concavity of the lumbosacral curve. Lateral stenosis was probably mostly due to degenerative phenomenon (disc, annulus, ligamentum flavum, facet) which generated both scoliosis and stenosis with specific and common symptoms (29). As previously demonstrated in Simmons' study with 30 patients and 
Epstein's study with 22 patients, central stenosis was not correlated to Cobb angle values and was rare in the concavity of the main curve, accordingly to prior reports $(7,30,31)$.

Radicular pain is one of the main complaints of ASD patients (7). Thus, in most of the cases, the goal of surgery is to relieve pain and to correct deformity. Correction-fusion could appear as the best treatment option. Nonetheless, due to patients' general health status, ASD correction surgery could be at risk due to his inherent complications and less invasive surgery could be considered. Therefore, if patient had numerous comorbidities, one option may be to perform limited decompression, relieving patients' radicular symptoms. That can be associated with a short fusion if there is spinal instability such as rotatory subluxation or spondylolisthesis, without global deformity correction.

\section{Limitations}

The main limitation of this study is that it is only a descriptive study of radio-anatomic parameters and clinical symptoms. The evaluation of surgical strategy according to this analysis would be interesting, since surgical management of these patients with both scoliosis and stenosis is challenging and since these patients often present comorbidities. The second limitation is that our findings are based on CT scan images. Previous reports have demonstrated that the diagnosis of foraminal stenosis with conventional imaging can be challenging (32). In the literature, CT was found to be more reliable than MRI given that multiplanar reconstructions were possible, whereas most of the time axial slices of MRI might have had different slice orientation from patient to patient and do not allow analysis in the plane of deformity $(16,33)$. Moreover, Ohba demonstrated the interest of CT images for the evaluation of foraminal stenosis in scoliotic patients (20). Obviously, we recommended the two to best understand the stenosis patterns. Another limitation was that the stenosis was not evaluated dynamically (flexion-extension views). In 2016, Merkle observed that LSS was misdiagnosed in 23\% of the supine MRI exams and highlighted the interest of dynamic myelography (34). More recently, Lau demonstrated that dynamic MRI in upright position improved the ability to find concordant radicular pain (35). Therefore, further investigations analyzing correlation between clinical symptoms of dynamic compression would be interesting. 


\section{Conclusion}

Lumbar spinal stenosis is frequent in ASD. Up to $72 \%$ of the patients suffered from severe central canal stenosis and were symptomatic in most of the cases. Foraminal and lateral stenosis were common (59\% and $58 \%$ of the patients, respectively). As a matter of fact, a precise evaluation of neurologic compression in scoliosis seems essential, particularly at the junction between main lumbar curve and lumbosacral contra-curve and the concavity of the lumbosacral contra-curve. Moreover, occurrence of L4L5 degenerative spondylolisthesis was always associated to central stenosis.

Therefore, concordance between clinical findings and radiographic exams is essential before surgical treatment. Nevertheless, the perfect planned treatment of stenosis and the correction of the scoliosis might not be always performed. It is a compromise most of the time, which is adapted to the patient symptoms and general health status. Thus, understanding the location of the symptomatic stenosis can help to direct appropriate surgical treatments for patients with lumbar scoliosis with associated stenosis. 


\section{Figures legends}

Figure 1: coronal and sagittal fullspine x-rays of a 66 years old woman with degenerative lumbar scoliosis $\left(41^{\circ}\right)$, L4L5 rotatory subluxation and lumbosacral contra-curve $\left(23^{\circ}\right)$.

Figure 2: coronal and sagittal x-rays with global alignment parameters (C7 coronal tilt on anterior and C7 sagittal tilt on lateral x-rays).

Figure 3: coronal x-rays, L3L4 open rotatory subluxation (1) and L3L4 closed rotatory subluxation (2).

Figure 4: stenosis measurements on CT scan images using multiplanar reconstructions, central canal stenosis on axial view (1), lateral recess stenosis on axial view (2), foraminal stenosis on sagittal view (3).

Figure 5: number of rotatory subluxations per patient.

Figure 6: distribution of central stenosis levels among patients.

Figure 7: coronal and sagittal fullspine x-rays of a 71 years old ASD patient (Cobb of $\left.27^{\circ}\right)$ with L3L4 and L4L5 stenosis.

Figure 8: axial (1), coronal (2) and sagittal (3) views of a 69 years old ASD patients (Cobb of $21^{\circ}$ ) with L3L4 rotatory subluxation and stenosis.

Figure 9: coronal and sagittal fullspine x-rays of a 61 years old ASD patient (Cobb of $31^{\circ}$ ) with a coronal tilt on the side of the concavity of the lumbosacral contra-curve. 


\section{References}

1. Schwab F, Farcy J-P, Bridwell K, et al. A clinical impact classification of scoliosis in the adult. Spine 2006 ;31(18):2109-14.

2. Schwab FJ, Smith VA, Biserni M, et al. Adult scoliosis: a quantitative radiographic and clinical analysis. Spine 2002;27(4):387-92.

3. Glassman SD, Berven S, Bridwell K, et al. Correlation of radiographic parameters and clinical symptoms in adult scoliosis. Spine 2005;30(6):682-8.

4. Glassman SD, Bridwell K, Dimar JR, et al. The impact of positive sagittal balance in adult spinal deformity. Spine 2005;30(18):2024-9.

5. Lafage V, Schwab F, Patel A, et al. Pelvic tilt and truncal inclination: two key radiographic parameters in the setting of adults with spinal deformity. Spine 2009 ;34(17):E599-606.

6. Ames CP, Smith JS, Scheer JK, et al. Impact of spinopelvic alignment on decision making in deformity surgery in adults: A review. J Neurosurg Spine 2012;16(6):547-64.

7. Morin C, Deburge A. [Lumbar stenosis with scoliosis. Symptomatologic study and surgical treatment of 39 cases]. Rev Chir Orthop Reparatrice Appar Mot. 1984;70(7):561-6.

8. Fu K-MG, Rhagavan P, Shaffrey CI, et al. Prevalence, severity, and impact of foraminal and canal stenosis among adults with degenerative scoliosis. Neurosurgery $2011 ; 69(6): 1181-7$.

9. Smith JS, Fu K-M, Urban P, et al. Neurological symptoms and deficits in adults with scoliosis who present to a surgical clinic: incidence and association with the choice of operative versus nonoperative management. J Neurosurg Spine 2008;9(4):326-31.

10. Grubb SA, Lipscomb HJ, Coonrad RW. Degenerative adult onset scoliosis. Spine 1988;13(3):241-5.

11. Faro FD, Marks MC, Pawelek J, et al. Evaluation of a functional position for lateral radiograph acquisition in adolescent idiopathic scoliosis. Spine 2004;29(20):2284-9.

12. Maillot C, Ferrero E, Fort D, et al. Reproducibility and repeatability of a new computerized software for sagittal spinopelvic and scoliosis curvature radiologic measurements: Keops(®). Eur Spine J Off Publ Eur Spine Soc Eur Spinal Deform Soc Eur Sect Cerv Spine Res Soc. 2015; 24(7):1574-81. 
13. Ferrero E, Ould-Slimane M, Gille O, et al. Sagittal spinopelvic alignment in 654 degenerative spondylolisthesis. Eur Spine J Off Publ Eur Spine Soc Eur Spinal Deform Soc Eur Sect Cerv Spine Res Soc. 2015; 24(6):1219-27.

14. Tassin JL, Guillaumat M, Piat L, et al. [Degenerative vertebral dislocation]. Ann Radiol (Paris). 1995;38(4):214-20.

15. Vialle R, Levassor N, Rillardon L, et al. Radiographic analysis of the sagittal alignment and balance of the spine in asymptomatic subjects. J Bone Joint Surg Am. 2005;87(2):260-7.

16. Waldt S, Gersing A, Brügel M. Measurements and classifications in spine imaging. Semin Musculoskelet Radiol. 2014;18(3):219-27.

17. Mamisch N, Brumann M, Hodler J, et al. Radiologic criteria for the diagnosis of spinal stenosis: results of a Delphi survey. Radiology. 2012;264(1):174-9.

18. Schonstrom NS, Bolender NF, Spengler DM. The pathomorphology of spinal stenosis as seen on CT scans of the lumbar spine. Spine. 1985;10(9):806-11.

19. Yamada K, Aota Y, Higashi T, et al. Roentgenographic and computed tomographic findings in symptomatic lumbar foraminal stenosis. Eur Spine J Off Publ Eur Spine Soc Eur Spinal Deform Soc Eur Sect Cerv Spine Res Soc. 2015;24(2):333-8.

20. Ohba T, Ebata S, Fujita K, et al. Characterization of symptomatic lumbar foraminal stenosis by conventional imaging. Eur Spine J Off Publ Eur Spine Soc Eur Spinal Deform Soc Eur Sect Cerv Spine Res Soc. 2015;24(10):2269-75.

21. Steurer J, Roner S, Gnannt R, et al. Quantitative radiologic criteria for the diagnosis of lumbar spinal stenosis: a systematic literature review. BMC Musculoskelet Disord. 2011;12:175.

22. Lee S, Lee JW, Yeom JS, et al. A practical MRI grading system for lumbar foraminal stenosis. AJR Am J Roentgenol. 2010;194(4):1095-8.

23. Mikhael MA, Ciric I, Tarkington JA, et al. Neuroradiological evaluation of lateral recess syndrome. Radiology. 1981;140(1):97-107.

24. Burton CV, Kirkaldy-Willis WH, Yong-Hing K, et al. Causes of failure of surgery on the lumbar spine. Clin Orthop. 1981;(157):191-9. 
25. Bao H, Yan P, Qiu Y, et al. Coronal imbalance in degenerative lumbar scoliosis: Prevalence and influence on surgical decision-making for spinal osteotomy. Bone Jt J. 2016;98-B(9):1227-33.

26. Ferrero E, Lafage R, Challier V, et al. Clinical and stereoradiographic analysis of adult spinal deformity with and without rotatory subluxation. Orthop Traumatol Surg Res OTSR. 2015;101(5):613-8.

27. Ferrero E, Lafage R, Diebo BG, et al. Tridimensional Analysis of Rotatory Subluxation and Sagittal Spinopelvic Alignment in the Setting of Adult Spinal Deformity. Spine Deform. 2017;5(4):255-64.

28. Aebi M. The adult scoliosis. Eur Spine J Off Publ Eur Spine Soc Eur Spinal Deform Soc Eur Sect Cerv Spine Res Soc. 2005;14(10):925-48.

29. Pritchett JW, Bortel DT. Degenerative symptomatic lumbar scoliosis. Spine. 1993;18(6):700-3.

30. Epstein JA, Epstein BS, Jones MD. Symptomatic lumbar scoliosis with degenerative changes in the elderly. Spine. 1979;4(6):542-7.

31. Simmons ED, Simmons EH. Spinal stenosis with scoliosis. Spine. 1992;17(6 Suppl):S117-120.

32. Infusa A, An HS, Glover JM, et al. The ideal amount of lumbar foraminal distraction for pedicle screw instrumentation. Spine. 1996;21(19):2218-23.

33. Attias N, Hayman A, Hipp JA, et al. Assessment of magnetic resonance imaging in the diagnosis of lumbar spine foraminal stenosis--a surgeon's perspective. J Spinal Disord Tech. 2006;19(4):249-56.

34. Merkle M, Maier G, Danz S, et al. The value of dynamic radiographic myelography in addition to magnetic resonance imaging in detection lumbar spinal canal stenosis: A prospective study. Clin Neurol Neurosurg. 2016;143:4-8.

35. Lau YYO, Lee RKL, Griffith JF, et al. Changes in dural sac caliber with standing MRI improve correlation with symptoms of lumbar spinal stenosis. Eur Spine J Off Publ Eur Spine Soc Eur Spinal Deform Soc Eur Sect Cerv Spine Res Soc. 2017 Jul 12; 


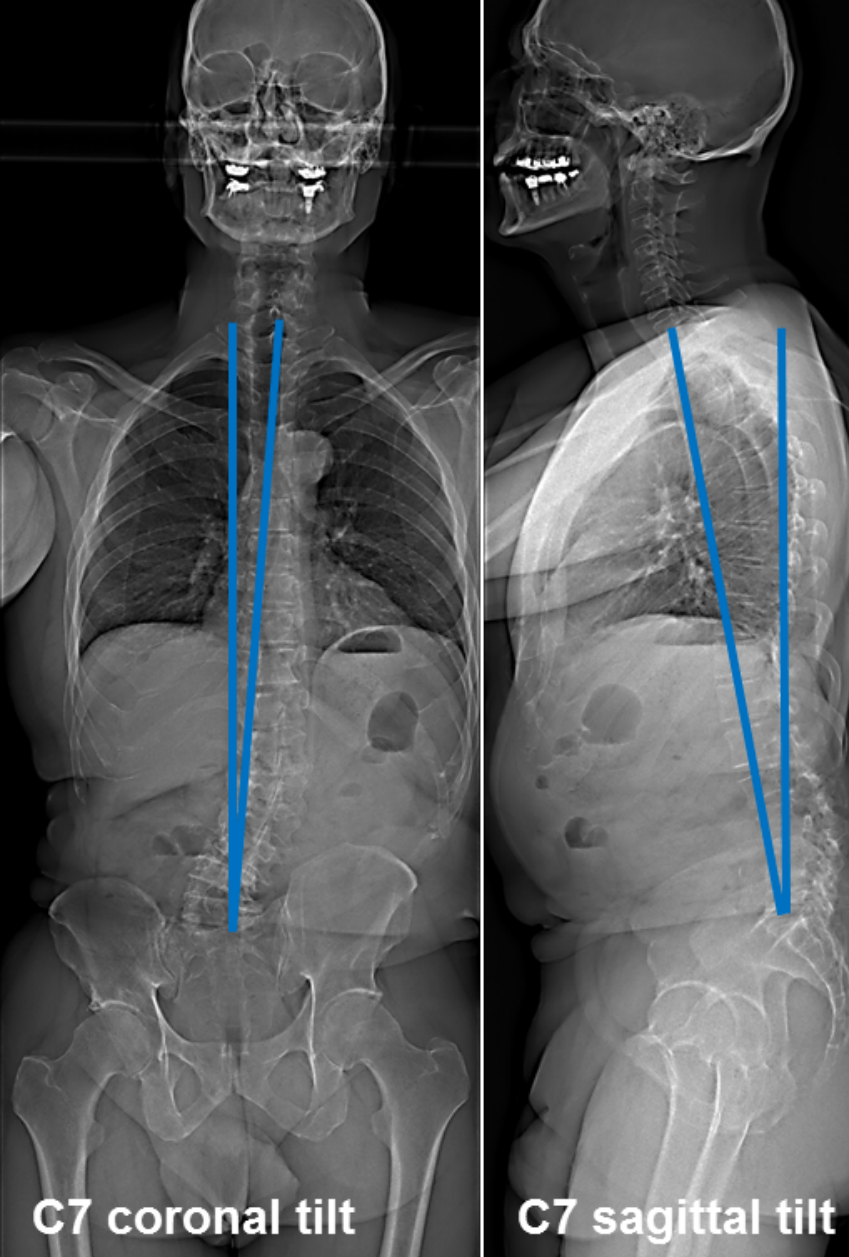


30

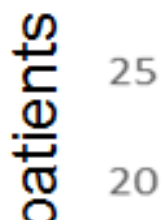

는 15

16

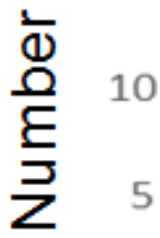

0

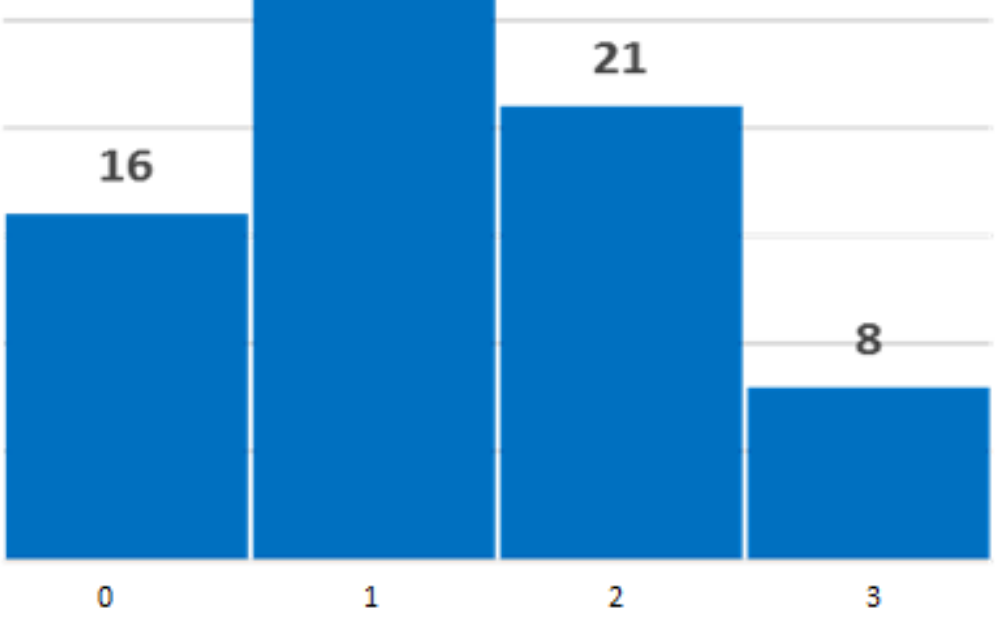

Number of rotatory subluxations 


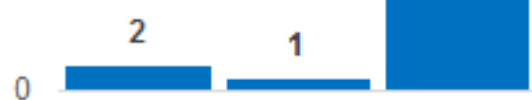

\section{$\begin{array}{lll}\text { T11T12 } & \text { T12L1 } & \text { L1L2 } \quad \text { L2L3 } \\ & \text { Stenotic levels }\end{array}$}

L3L4 L4L5 L5S1

L3L4 L4L5 L5S1 


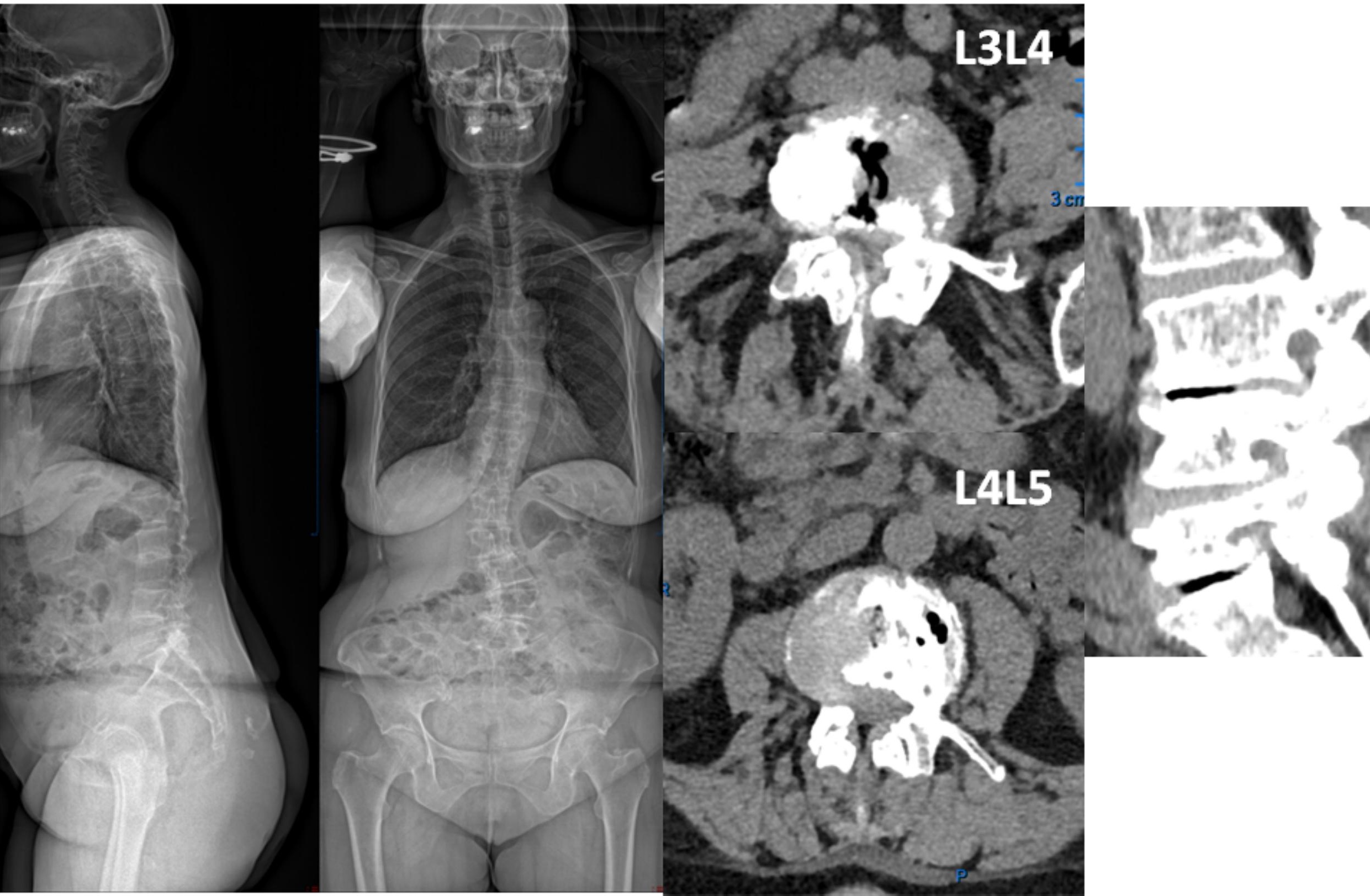




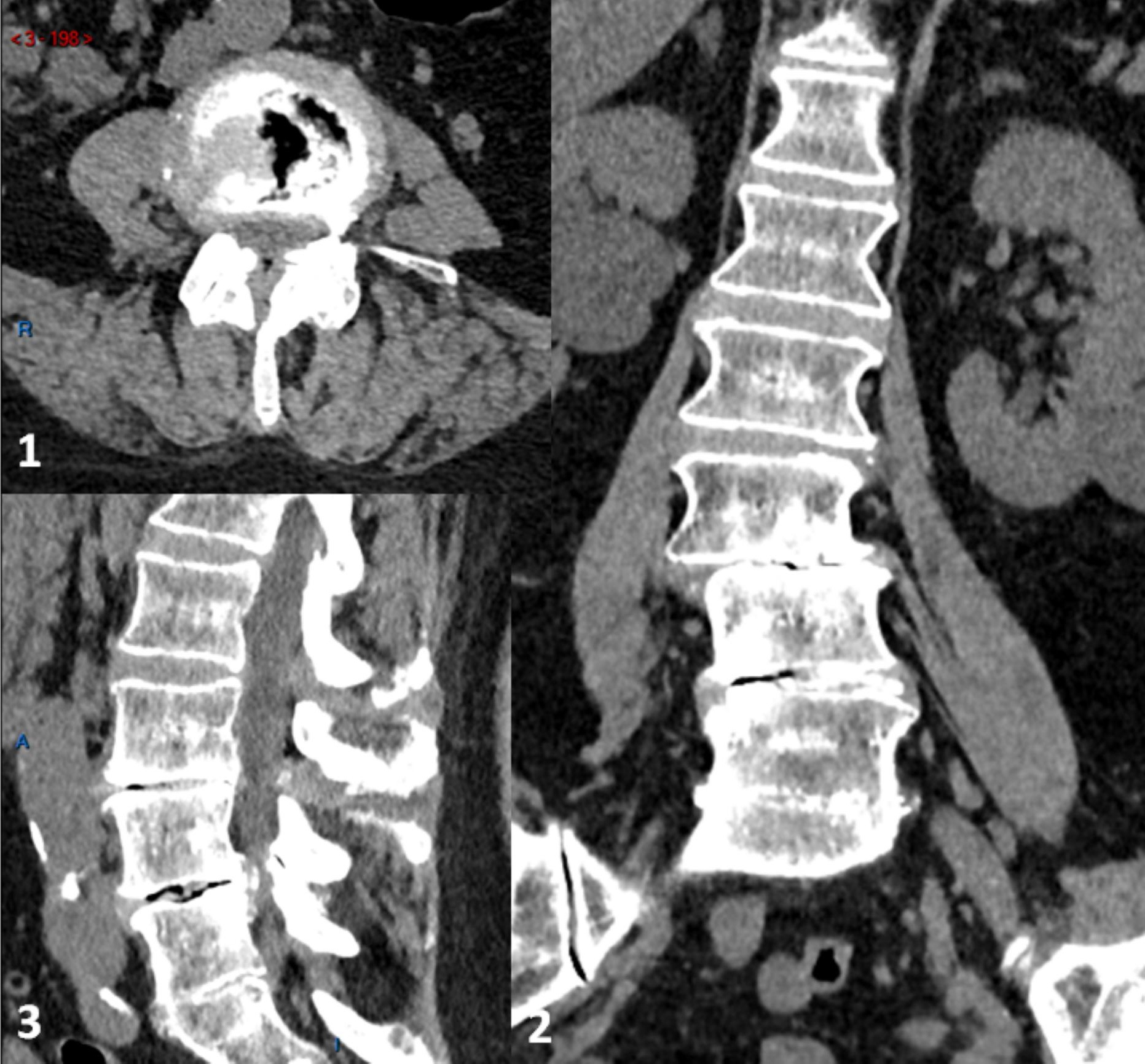


Table 1: spinopelvic parameters of the entire cohort

\begin{tabular}{l|cccc} 
& Mean & SD & Minimum & Maximum \\
\hline \hline C7 sagittal tilt $\left(^{\circ}\right)$ & 6,1 & 5,5 & -4 & 23,9 \\
Maximal thoracic kyphosis $\left(^{\circ}\right)$ & 44 & 15 & 15 & 67 \\
Maximal lumbar lordosis $\left(^{\circ}\right)$ & 42 & 18 & -6 & 73 \\
Pelvic incidence $\left(^{\circ}\right)$ & 56 & 14 & 34 & 91 \\
Pelvic tilt $\left(^{\circ}\right)$ & 24 & 10 & 1 & 48 \\
Sacral slope $\left({ }^{\circ}\right)$ & 32 & 12 & 2 & 63
\end{tabular}

Table 2: values of central surface area for central stenosis measurement. SD: standard deviation.

\begin{tabular}{l|cccc} 
& & & & \\
Vertebral levels & Mean & SD & minimum & maximum \\
\hline \hline T11T12 $\left(\mathbf{m m}^{\mathbf{2}}\right)$ & 176 & 46 & 78 & 280 \\
T12L1 $\left(\mathbf{m m}^{\mathbf{2}}\right)$ & 182 & 43 & 78 & 283 \\
L1L2 $\left(\mathbf{m m}^{\mathbf{2}}\right)$ & 148 & 37 & 60 & 233 \\
L2L3 $\left(\mathbf{m m}^{\mathbf{2}}\right)$ & 108 & 42 & 35 & 243 \\
L3L4 $\left(\mathbf{m m}^{\mathbf{2}}\right)$ & 85 & 36 & 27 & 206 \\
L4L5 $\left(\mathbf{m m}^{\mathbf{2}}\right)$ & 76 & 45 & 21 & 193 \\
L5S1 $\left(\mathbf{m m}^{\mathbf{2}}\right)$ & 134 & 56 & 40 & 266
\end{tabular}

Table 3: values of foramen stenosis (diameter in $\mathrm{mm}$ ).

SD: standard deviation

\begin{tabular}{l|cccc} 
& \multicolumn{2}{|c}{ right foramen } & \multicolumn{2}{c}{ left foramen } \\
& Mean & SD & Mean & SD \\
\hline \hline L1 height & 10,9 & 2,7 & 9,9 & 2,6 \\
L1 width & 6,1 & 1,4 & 6,4 & 1,7 \\
L2 height & 9,6 & 2,6 & 8,7 & 2,9 \\
L2 width & 5,6 & 1,6 & 5,7 & 1,8 \\
L3 height & 8,7 & 2,8 & 9,0 & 3,3 \\
L3 width & 5,9 & 1,7 & 5,9 & 1,5 \\
L4 height & 7,8 & 2,3 & 8,8 & 2,4 \\
L4 width & 6,0 & 1,4 & 6,2 & 1,6 \\
L5 height & 7,1 & 2,3 & 7,5 & 2,3 \\
L5 width & 6,7 & 1,9 & 6,3 & 1,5
\end{tabular}


Table 4: values of lateral recess stenosis (diameter in $\mathrm{mm}$ ).

$S D$ : standard deviation

\begin{tabular}{l|cccc} 
& \multicolumn{2}{|c}{ right } & \multicolumn{2}{c}{ left } \\
& Mean & SD & Mean & SD \\
\hline \hline L3 & 5,3 & 1,2 & 5,4 & 1,0 \\
L4 & 4,7 & 1,0 & 4,8 & 1,0 \\
L5 & 3,8 & 1,2 & 4,0 & 1,0
\end{tabular}

